\title{
Papillary Thyroid Carcinoma Associated with Caseating Granulomatous Inflammation: A Case Report from United Arab Emirates
}

\author{
Sreekumar Rajakumar*, Noureddine Al Hasan and Mohammed Mater Al Falasi \\ Department of ENT, Tawam Hospital, Al Ain, Abu Dhabi, United Arab Emirates
}

\begin{abstract}
The study presents the case of a 37-year-old female expatriate who was referred with a neck swelling and was managed further in Tawam Hospital, Al Ain. Initial ultrasonography and fine needle aspiration cytology from the right thyroid nodule confirmed papillary carcinoma of thyroid Bethesda type 6 and fine needle aspiration cytology from the right mid jugular lymph node proved metastasis from the primary. Further evaluation by CT scan neck with contrast and a total thyroidectomy and bilateral selective neck dissection was done. Histopathology confirmed classic papillary thyroid carcinoma with metastasis and caseating granulomatous inflammation in a section of thyroid and few lymph nodes. This case report is a rare presentation of papillary thyroid carcinoma with caseating granulomatous inflammation and presents a challenge for further management and a possible role of caseating granulomatous infection in the tumorigenesis of Papillary thyroid carcinoma.
\end{abstract}

\section{Keywords}

Papillary thyroid carcinoma, Thyroidectomy, Caseating granulomatous inflammation

\section{Introduction}

Thyroid cancer is the most common endocrine malignancy and clinical thyroid cancer accounts for $1 \%$ to $2 \%$ of all cancers [1]. In the UAE, thyroid cancer is the sixth most cancer and the second among females [2]. Papillary thyroid carcinoma is the most common thyroid cancer and the standard of care has already been decided based on tumour site, size, lymph node and distant metastasis [3]. Total thyroidectomy is performed in most cases and [4] additional neck dissection is performed if metastasis is confirmed by ultrasonography, CT scan, FNAC. The mortality rate associated with thyroid cancer is only $1.35 / 1000000$ people and therefore unnecessary resection should be avoided.

UAE population includes local Emiratis and expatriates. Expatriate population mainly includes Indians, Pakistanis, Bangladeshis, Filipinos, rest of middle east and far east Arabs, Europeans and Americans [5]. Incidence of caseating granulomatous inflammation is very high among Asians and the presentation of metastatic lymph nodes from papillary thyroid carcinoma emphasizes the co-existence of caseating granulomatous inflammation in patients with papillary thyroid carcinoma.

\section{Presentation}

A 37-year-old female (Philippine expatriate) was referred to the ENT outpatient clinic with a neck swelling of more than
6 months duration. She had no associated complaints of recurrent fever, loss of weight, loss of appetite, change in voice or difficulty in swallowing. Before referral she was evaluated in a private clinic with ultrasonography and FNAC from the right thyroid nodule and right mid jugular lymph node which confirmed papillary thyroid carcinoma and metastasis. A CT scan neck with contrast was done in Tawam Hospital (Figure 1 and Figure 2).

A total thyroidectomy and bilateral selective neck dissection was done. Under general anaesthesia with neuromonitoring, apron skin incision, subplatysmal flap raised, thyroid gland with strap muscles exposed, total thyroidectomy done with careful preservation of bilateral recurrent laryngeal nerve, thyroid gland shaved off from the trachea (Figure 3 and Figure 4). Bilateral central compartment neck dissection

*Corresponding author: Dr. Sreekumar Rajakumar, Department of ENT, Tawam Hospital, Al Ain, Abu Dhabi, United Arab Emirates

Accepted: September 26, 2020

Published online: September 28, 2020

Citation: Rajakumar S, Al Hasan N, Al Falasi MM (2020) Papillary Thyroid Carcinoma Associated with Caseating Granulomatous Inflammation: A Case Report from United Arab Emirates. J Head Neck Surg 2(1):66-69 
Citation: Rajakumar S, Al Hasan N, Al Falasi MM (2020) Papillary Thyroid Carcinoma Associated with Caseating Granulomatous Inflammation: A Case Report from United Arab Emirates. J Head Neck Surg 2(1):66-69

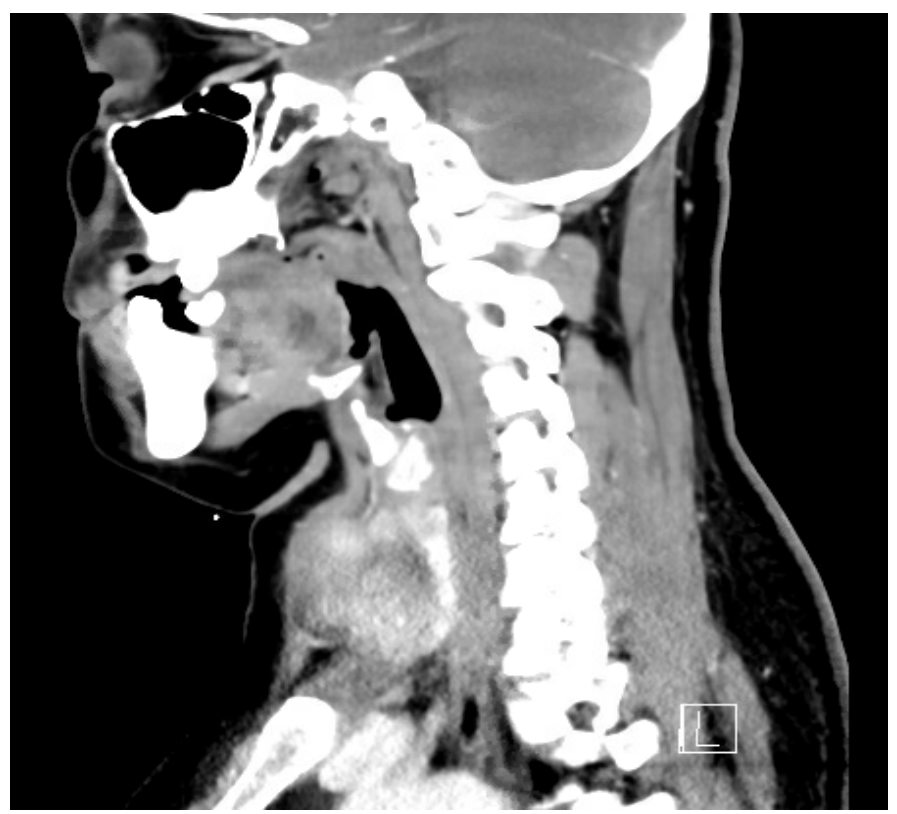

Figure 1

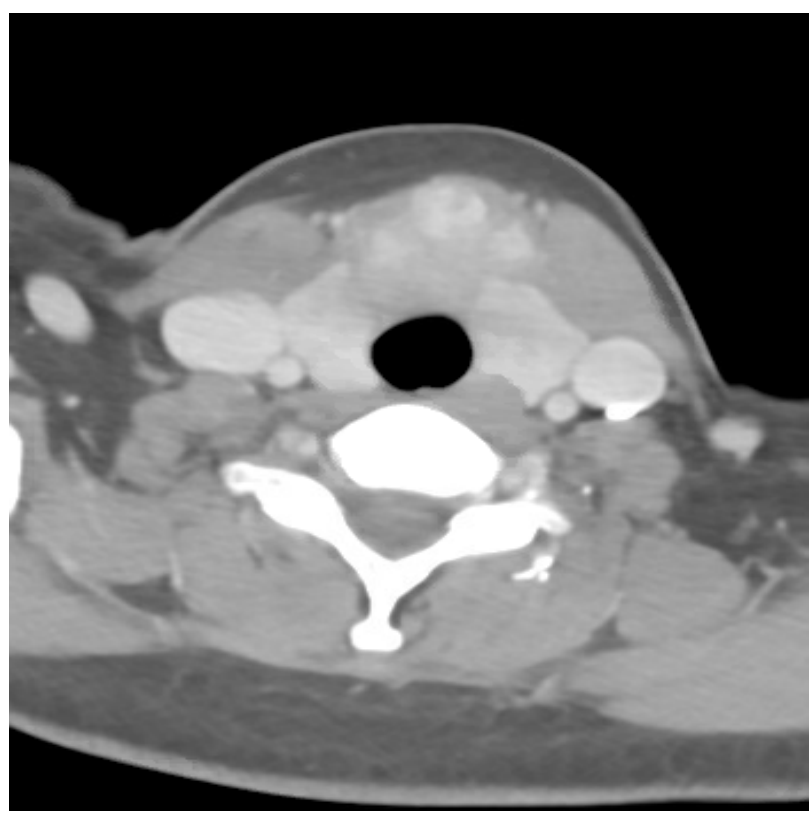

Figure 2

Figure 1 and Figure 2: Markedly heterogeneously enhancing solid looking lobulated mass occupying the isthmus, right and left lobe of thyroid inferiorly and measures $5 \mathrm{~cm} \times 2.3 \mathrm{~cm} \times 4.4 \mathrm{~cm}$ with tiny specks of calcification. The mass also flattens anterior wall of trachea although no frank internal extension visualized. Suspicious lymph nodes adjacent to the right internal jugular vein, left carotid vessel, right supraclavicular region, left suprasternal notch visualized.

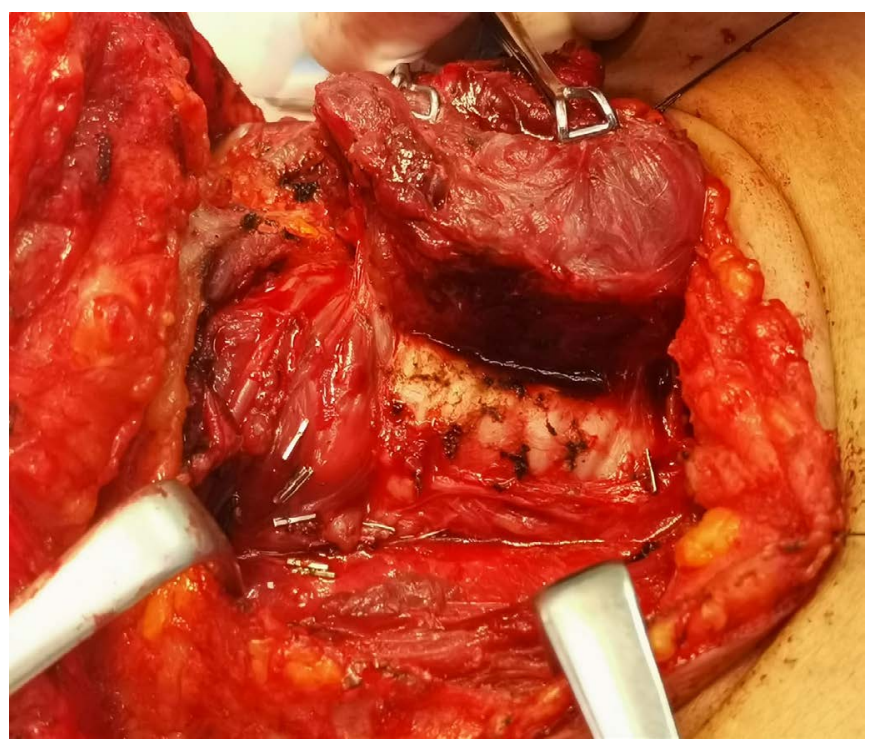

Figure 3

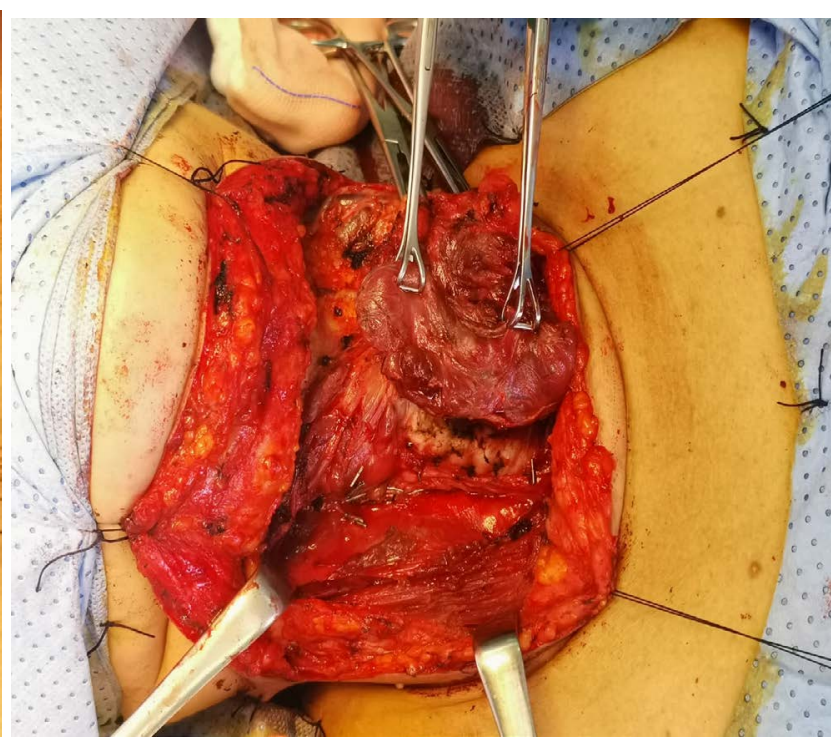

Figure 4

Figure 3 and Figure 4: Total thyroidectomy with careful preservation of bilateral recurrent laryngeal nerve, thyroid gland shaved off from the trachea.

done followed by bilateral selective dissection of levels 2, 3, 4, 5 preserving sternocleidomastoid, internal jugular vein and spinal accessory nerve done. Post-operative period was uneventful.

Pathology report confirmed classic (conventional) papillary thyroid carcinoma with vascular and lymphatic invasion and presence of extra thyroidal extension. Of the 93 regional lymph nodes examined, 21 lymph nodes were positive for metastasis with the largest metastatic deposit of $2 \mathrm{~cm}$ and extra nodal extension present. (PT3b PN1b) There were several lymph nodes involved by caseating granulomatous inflammation which was also noted in a section of thyroid gland (Figure 5 and Figure 6).

With the diagnosis of papillary thyroid carcinoma, metastasis and caseating granulomatous inflammation, infectious medicine was consulted. Patient was started empirically on 

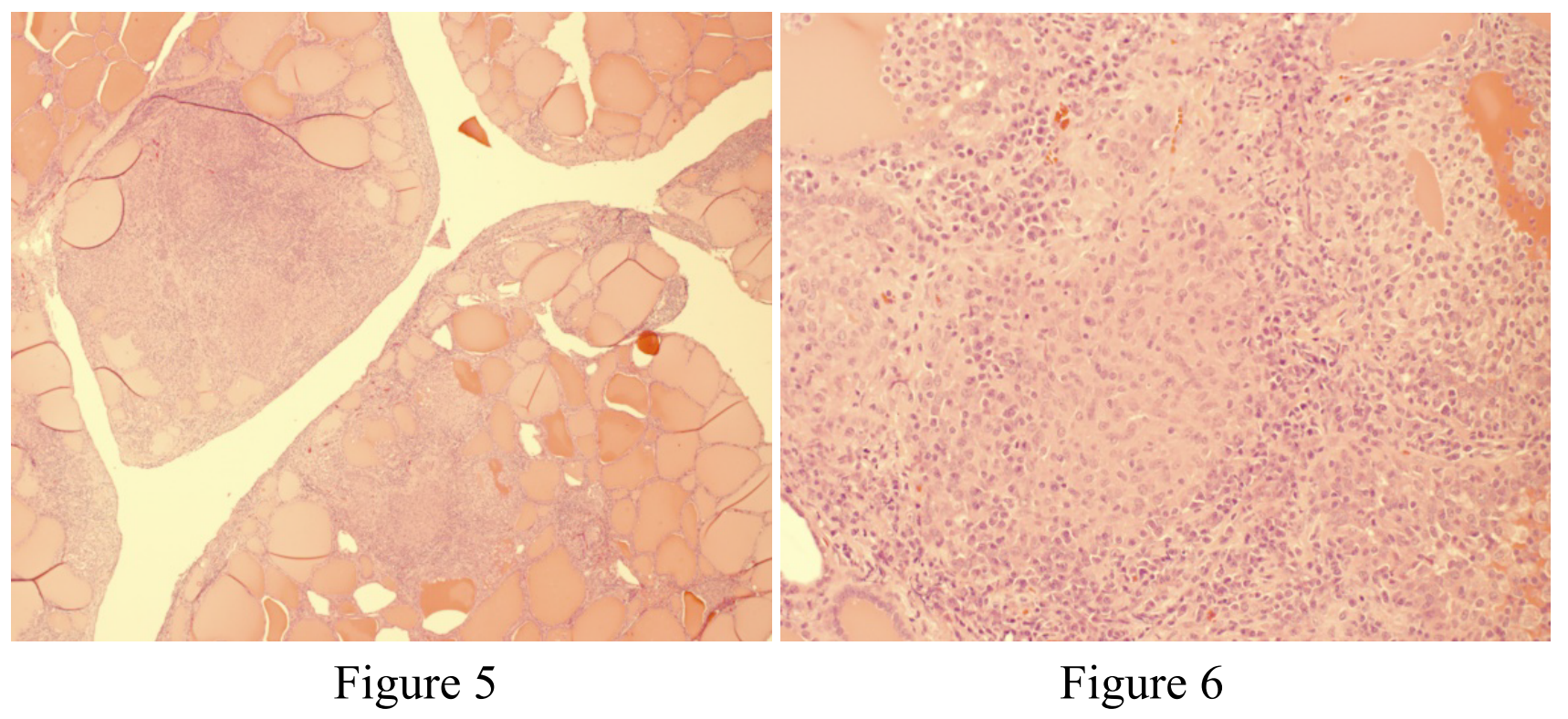

Figure 5 and Figure 6: Thyroid section. Caeseating granuloma.

anti tuberculosis therapy with future plan for radioactive iodine ablation.

\section{Discussion}

Thyroid gland tuberculosis is a rare disease with a frequency of $0.1 \%$ to $0.4 \%$ in histologically diagnosed thyroid specimens [6]. Even in Asian countries where the prevalence of tuberculosis is high, the incidence of thyroid tuberculosis remains low. It is very difficult to diagnose thyroid TB due to lack of specific symptoms or signs. It can present as an isolated nodule, multinodular goitre, abscess or chronic skin sinus [7]. Imaging technique is not very useful in the diagnosis [8]. FNAC with AFB is the best method for the diagnosis of thyroid tuberculosis preoperatively [9]. However majority of cases are diagnosed based on post-operative histopathology. In this case, the presence of caseating granulomatous inflammation in the thyroid section and the lymph nodes supported the final diagnosis [10]. Association of papillary thyroid carcinoma or the development of PTC with caseating granulomatous inflammation maybe due to chronic inflammation [11]. Caseating granulomas establish persistent inflammation with previous studies reporting DNA damage through production of nitric oxide and reactive oxygen species [12]. This DNA damage has been implicated in inflammation related carcinogenesis [13]. Associated antiapoptotic activity through upregulation of B-cell lymphoma-2 gene expression [14,15], elevated concentration of prostaglandins following caseating granulomatous inflammation. Combination of DNA damage, apoptosis inhibition, chronic inflammation may result in tumorigenesis. Presence of caseating granulomas in lymph nodes may result in over staging when using the TNM system [16]. It's mentioned that preoperative lymphatic puncture effectively prevents over staging and excessive surgery. In conclusion, this is a rare presentation of coexisting papillary thyroid carcinoma and caseating granulomatous inflammation and implicated the possible rule for tumorigenesis.

\section{References}

1. Nabil Al-Zaher, Suhail Al-Salam, Hassan El Teraifi (2008) Thyroid carcinoma in the united Arab Emirates: Perspectives and experience of a tertiary care hospital. Hematology/Oncology and Stem Cell Therapy 1: 14-21.

2. Liwei Meng, Shan Hu, Liming Huang, et al. (2014) Papillary thyroid cancer coexisting with thyroid tuberculosis: A case report. Oncology Letters 7: 1563-1565.

3. Mayara Peres Barbosa, DeniseMomesso, Daniel Alves Bulzico, et al. (2017) Metastatic lymph node characteristics as predictors of recurrence/persistence in the neck and distant metastasis in differentiated thyroid cancer. Arch Endocrinol Metab 61: 6.

4. Yasuhiro Ito, Akira Miyauchi, Takumi Kudo, et al. (2017) The effectiveness of prophylactic modified neck dissection for reducing the development of lymph node recurrence of papillary thyroid carcinoma. World J Surg 41: 2283-2289.

5. Suri VS, Sakhuja P, Malhotra V, et al. (2002) Co-existent tuberculosis and papillary carcinoma thyroid. Trop Doct 32: 118.

6. Bulbuloglu E, Ciralik H, Okur E, et al. (2006) Tuberculosis of the thyroid gland: Review of the literature. World J Surg 30: 149-155.

7. Ghosh A, Saha S, Bhattacharya B, et al. (2007) Primary tuberculosis of thyroid gland: A rare case report. Am J Otolaryngology 28: 267-270.

8. Kang M, Ojili V, Khandelwal N, et al. (2006) Tuberculous abscess of the thyroid gland: A report of two cases. J Clin Ultrasound 34: 254-257.

9. Goel MM, Budhwar P (2008) Fine needle aspiration cytology and immunocytochemistry in tuberculous thyroiditis: A case report. Acta Cytol 52: 602-606.

10. Majid U, Islam N (2011) Thyroid tuberculosis: A case series and a review of the literature. J Thyroid Res.

11. Schottenfeld D, Beebe-Dimmer J (2006) Chronic inflammation: A common and important factor in the pathogenesis of neoplasia. CA Cancer J Clin 56: 69-83. 
12. Falagas ME, Kouranos VD, Athanassa Z, et al. (2010) Tuberculosis and malignancy. QJM 103: 461-487.

13. Kawanishi S, Hiraku Y, Pinlaor S, et al. (2006) Oxidative and nitrative DNA damage in animals and patients with inflammatory diseases in relation to inflammation-related carcinogenesis. Bio Chem 387: 365-372.

14. Zhang J, Jiang R, Takayama $\mathrm{H}$, et al. (2005) Survival of virulent Mycobacterium tuberculosis involves preventing apoptosis induced by $\mathrm{Bcl}-2$ upregulation and release resulting from necrosis in J774 macrophages. Microbiol Immunol 49: 845-852.
15. Rangel Moreno J, Estrada García I, De La Luz García Hernández $M$, et al. (2002) The role of prostaglandin E2 in the immunopathogenesis of experimental pulmonary tuberculosis. Immunology 106: 257-266.

16. Taku Ito, Hiroaki Saito, Naomi Ki shine, et al. (2015) Preoperatively diagnosed case with co-existence of papillary thyroid carcinoma and cervical tuberculous lymphadenitis. International Journal of Surgery Case Reports 15: 74-77. 